\title{
Highly Sensitive Gate/Body-Tied Metal-Oxide- Semiconductor Field-Effect Transistor-Type Photodetector with Wavelength-Selective Metal Grid Structure Using Standard Complementary Metal-Oxide-Semiconductor Technology
}

\author{
Hee Ho Lee, Sung-Hyun Jo, Myunghan Bae, Byung-Soo Choi, \\ Jeongyeob Kim ${ }^{1}$, Hong-Kun Lyu ${ }^{2}$ and Jang-Kyoo Shin* \\ School of Electronics Engineering, Kyungpook National University, \\ 80 Daehakro, Bukgu, Daegu 702-701, Korea \\ 'Department of Sensor and Display Engineering, Kyungpook National University, \\ 80 Daehakro, Bukgu, Daegu 702-701, Korea \\ ${ }^{2}$ IT Convergence Research Division, Daegu Gyeongbuk Institute of Science and Technology, \\ 333, Techno jungang-daero, Hyeonpung-myeon, Dalseong-gun, Daegu 711-873, Korea
}

(Received July 18, 2014; accepted December 8, 2014)

Key words: gate/body-tied MOSFET-type photodetector, metal grid, wavelength selectivity, high sensitivity

In this research, a highly sensitive gate/body-tied metal-oxide-semiconductor fieldeffect transistor (MOSFET)-type photodetector with a wavelength-selective metal grid structure was designed and fabricated using $0.18-\mu \mathrm{m}$ standard complementary metaloxide-semiconductor technology. This device is composed of a floating gate that is tied to a well and a wavelength-selective metal grid is placed on top of each photodetector. The designed metal grid structure included one-dimensional and two-dimensional patterned metal layers. The amplified photocurrent of the gate/body-tied MOSFETtype photodetector was found to be more than 1000-fold that of a conventional $n+/ p$-sub photodiode with the same area. To demonstrate the wavelength selectivity, we measured the drain current and transmittance of the photodetector as a function of wavelength.

\section{Introduction}

Traditionally, in color image capture technology, color filter arrays (CFAs) inserted in the optical path have been used to control the transmittances of different wavelengths. ${ }^{(1,2)}$ There are two main types of CFAs: 1) Bayer pattern, which uses the primary colors red, green, and blue (RGB), and 2) complementary color scheme, which uses cyan, magenta,

"Corresponding author: e-mail: jkshin@ee.knu.ac.kr 
and yellow (CMY). ${ }^{(3,4)}$ However, these conventional dye-doped color filters pose some problems such as color cross talk and additional postprocessing costs. ${ }^{(5)}$ Usually, the color filters account for a major part of the manufacturing cost of a sensing system. Considering the trend to move toward complementary metal-oxide-semiconductor (CMOS)-based photodetectors, it is desirable to implement a fully integrated architecture that integrates color filters into the detectors. ${ }^{(1,6)}$

A p-n junction photodiode is commonly used as a photodetector in CMOS image sensors. However, a standard silicon photodiode has poor sensitivity and produces large ripples because of a small photocurrent. (7) Another disadvantage of photodiode-based CMOS sensors is that the amplitude of the output signal decreases when the sensor dimensions are reduced. However, metal-oxide-semiconductor field-effect transistor (MOSFET)-based sensors do not have these problems. ${ }^{(8)}$

In this paper, we propose a combination of a highly sensitive gate/body-tied MOSFET-type photodetector with a wavelength-selective metal grid pattern. The proposed photodetector was fabricated using a commercial $0.18-\mu \mathrm{m}$ CMOS process, without postprocessing. One-dimensional (1D) and two-dimensional (2D) metal grid patterns with minimum width and different periodicities were fabricated using already available metal layers by a standard process. The photodetectors were illuminated by monochromatic light within the visible range. The output photocurrents of the detectors were measured for incident wavelengths ranging from 400 to $700 \mathrm{~nm}$, with a step of 20 $\mathrm{nm}$. The spectral transmittances were evaluated using the ratio of the output current of each photodetector to the output current of the uncovered reference photodetector.

\section{Experiments}

\subsection{Gate/body-tied MOSFET-type photodetector}

Figure 1 shows the cross-sectional view and the vertical energy band diagram of the gate/body-tied MOSFET-type photodetector. This sensor was originally proposed by Zang and $\mathrm{Chan}^{(9)}$ The photodetector consists of a p-channel MOSFET (PMOSFET), with a floating gate tied to an n-well, and is fabricated by a $0.18-\mu \mathrm{m}$ standard CMOS process. The PMOSFET is actually a buried channel device to obtain the desired threshold voltage attributable to the $\mathrm{n}+$ polysilicon gate used. The Fermi level of an $\mathrm{n}$-well differs from that of an $n+$ polysilicon gate. This causes band bending in the upper region of the buried channel, and the height of the energy barrier from the level at the $\mathrm{Si} / \mathrm{SiO}_{2}$ interface is nearly equal to the difference in the Fermi level between the $n+$ polysilicon and the $n+$ diffusion outlet. ${ }^{(8)}$

The basic operational principle of the MOSFET-type photodetector is as follows. ${ }^{(9)}$ When the device is illuminated, the incoming photons generate electron-hole pairs. The photogenerated electron-hole pairs are separated by a vertical field, which is produced by band bending (Fig. 1) mentioned earlier. The generated holes move through the channel to the drain, owing to the applied drain bias voltage. On the other hand, the generated electrons move away to the bottom of the n-well where they accumulate owing to their potential barrier. ${ }^{(9,10)}$ These accumulated electrons induce a negative bias to the n-well, which is fed back to the gate through the gate/n-well connection. Owing to this positive 


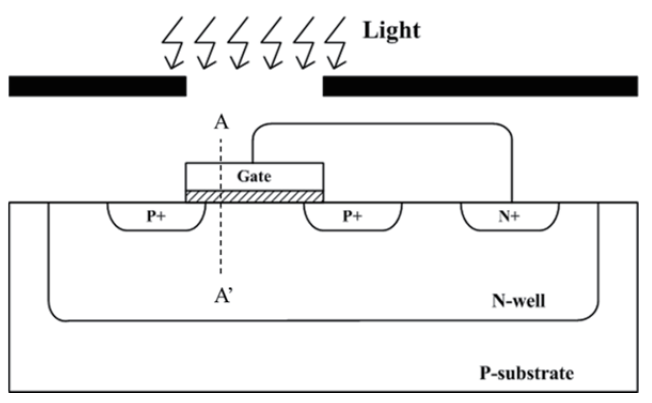

(a)

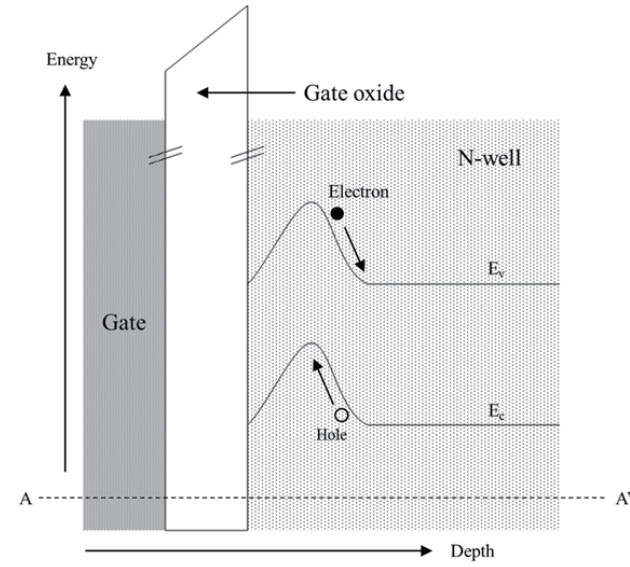

(b)

Fig. 1. Gate/body-tied MOSFET-type photodetector: (a) cross-sectional view and (b) vertical energy band diagram.

feedback mechanism, the photocurrent is highly amplified. Therefore, the MOSFETtype photodetector, which has a high sensitivity, can be used to obtain an amplified photocurrent.

\subsection{Wavelength-selective metal grid pattern}

Ghaemi et al. investigated a periodic subwavelength metal grid array in a silver film and observed the grid's wavelength-filtering properties. ${ }^{(11)}$ The transmission spectrum of the metal grid in a square lattice, at normal incidence, is related to the period of the array, the dielectric constants of the metal, and the dielectric material in contact with the metal. ${ }^{(12)}$

The structure of the gate/body-tied MOSFET-type photodetector with a metal grid pattern is shown in Fig. 2. The designed metal grid structure includes 1D and 2D patterned metal layers placed on top of the gate/body-tied MOSFET-type photodetector (Fig. 3). These patterns have periodicities ranging from 540 to $720 \mathrm{~nm}$ and spacings ranging from 270 to $450 \mathrm{~nm}$. Moreover, the designed metal grid pattern is made of 500-nmthick aluminum, which is commonly used in the standard CMOS process. The metal-1 layer, which is the closest to the photodetector from the metal line, was chosen because placing the light filters closer to the photodetector reduces color cross-talk and pixel vignetting. ${ }^{(1)}$ As shown in Fig. 3, it can be confirmed that the actual patterns were close to the design specifications.

The area of the fabricated photodetector is $7.3 \times 7.6 \mu \mathrm{m}^{2}$. A 3.3-V, 7.6-nm-thick oxide transistor was used to increase the voltage swing and reduce the transistor gate leakage. We included an uncovered reference pixel on the chip because the transmittance spectrum of the photodetector is not directly measured. The transmittance of each photodetector was measured by normalizing the responsivity of each photodetector, using the responsivity of the uncovered reference photodetector. 

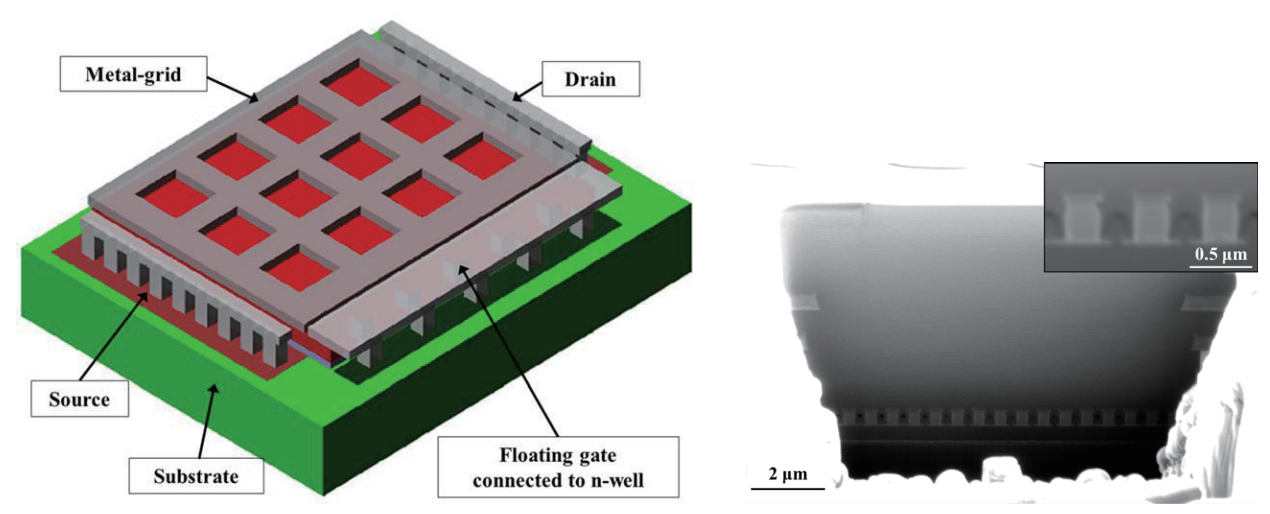

Fig. 2 (left). (Color online) Structure of gate/body-tied MOSFET-type photodetector with metal grid pattern.

Fig. 3 (right). Scanning electron microscopy images of metal grid pattern in $0.18-\mu \mathrm{m}$ standard CMOS technology.

\section{Results and Discussion}

We designed and fabricated a highly sensitive gate/body-tied MOSFET-type photodetector with a wavelength-selective metal grid pattern, using a $0.18-\mu \mathrm{m}$ standard CMOS process, and investigated its use in color imaging. The fabricated chip, shown in Fig. 4, is an array of gate/body-tied photodetectors with metal grid structures. The output of each photodetector is connected to a pin for testing. We also included a gate/ body-tied photodetector with no grids for the purpose of comparison.

To test the designed sensor, a Thermo Oriel 74000 monochromator was used to generate monochromatic light, with a Driel 66000 lamp as a white light source. The output wavelength of the monochromator was controlled by a LabVIEW program via an RS-232 port. A wavelength sweep, from 400 to $700 \mathrm{~nm}$ with steps of $20 \mathrm{~nm}$, was performed. The electrical characteristics of the designed sensor were measured using an Agilent 4156 semiconductor parameter analyzer.

Figure 5 shows the $I-V$ characteristics of the gate/body-tied MOSFET-type photodetector under several illumination conditions, from dark to 30000 lux. The characteristics of the photodetector were measured in the common drain mode with a different illumination level. A halogen lamp was used as the light source in this experiment. The channel potential of the photodetector varies according to the incident light intensity. This channel potential variation induces a current variation in the photodetector. $^{(9)}$

A comparison of the responsivity per unit area for the conventional $n+/ p$-sub photodiode with the gate/body-tied MOSFET-type photodetector, using the same $0.18-\mu \mathrm{m}$ CMOS fabrication process, is shown in Fig. 6. As shown in the figure, the responsivity of the gate/body-tied MOSFET-type photodetector is more than 1000-fold 


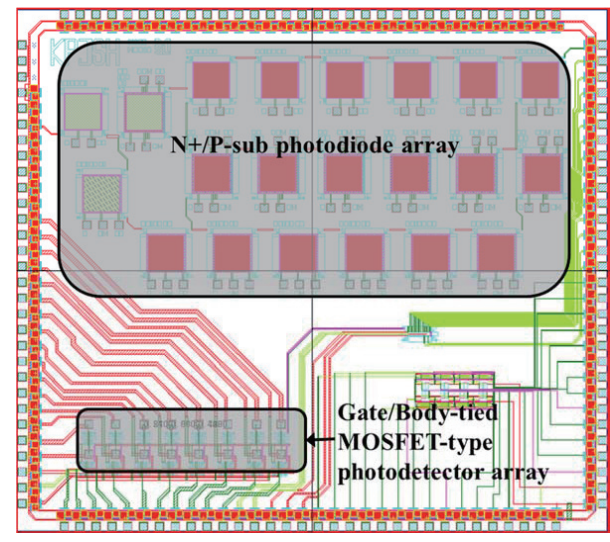

(a)

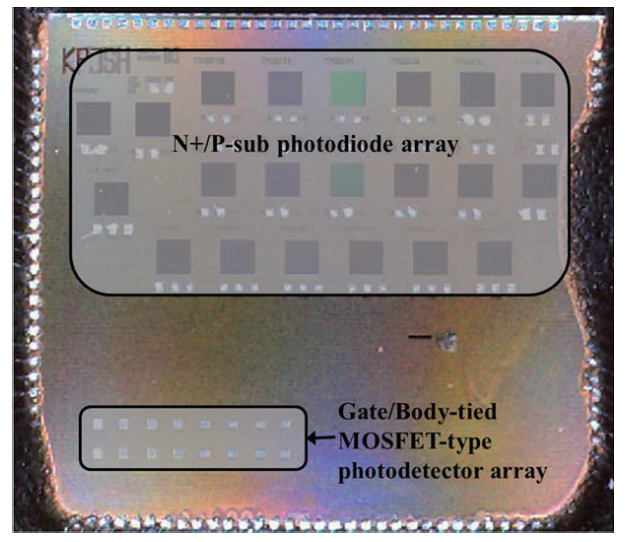

(b)

Fig. 4. (Color online) (a) Layout and (b) photograph of fabricated chip.
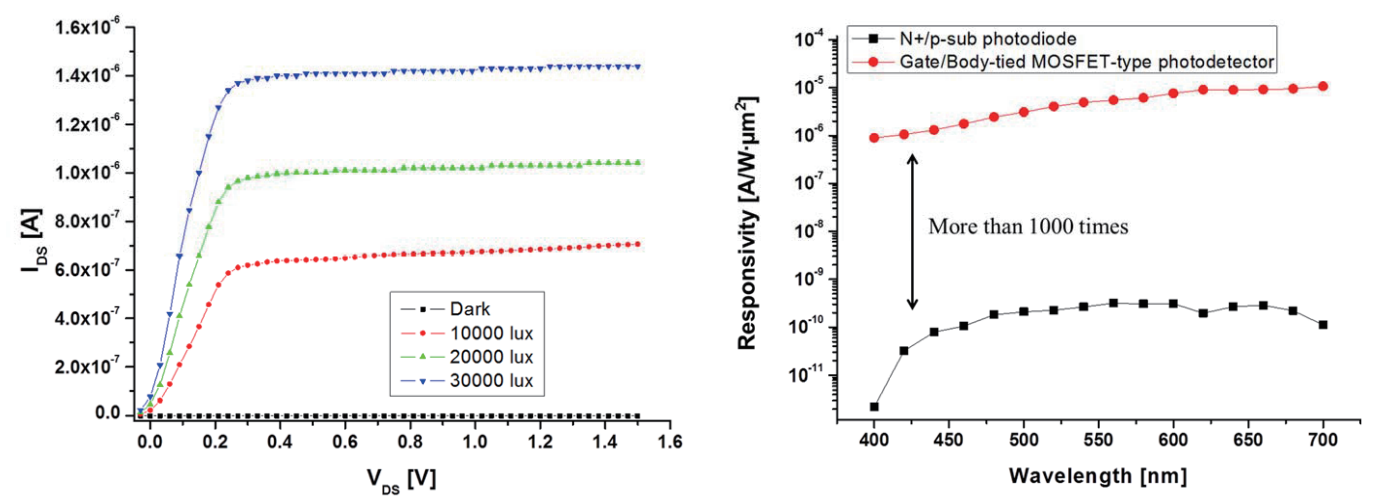

Fig. 5 (left). (Color online) Optical characteristics of gate/body-tied MOSFET-type photodetector. Fig. 6 (right). (Color online) Measurement results of variation in responsivity with wavelength.

that of the conventional $\mathrm{n}+/ \mathrm{p}$-sub photodiode for wavelengths from 400 to $700 \mathrm{~nm}$. This result confirms that the gate/body-tied MOSFET-type photodetector not only has better performance even though it occupies a smaller area, but it can also overcome signal reduction according to the metal grid pattern. ${ }^{(1,6)}$

The spectral drain current and transmittance measurement results for the gate/bodytied photodetectors with the 1D metal grid and with unpolarized illumination are shown in Fig. 7. The transmittance of the gate/body-tied photodetector with the 1D metal grid increases when the period/spacing of the metal grid increases. The spectral drain current 
and transmittance measurement results for the gate/body-tied photodetectors with the 2D metal grid and with unpolarized illumination are shown in Fig. 8. Similar to the results obtained for the 1D metal grid, the transmittance of the gate/body-tied photodetectors with the 2D metal grid increases when the period/spacing of the metal grid increases. The wavelength is smaller than the designed metal grid; hence, the transmittance can be explained by the vectorial diffraction theory. ${ }^{(13)}$ The wavelength is larger than the designed metal grid; hence, surface plasmon resonances are present. ${ }^{(14)}$ Therefore, the transmittance of the proposed photodetector shows significant wavelength selectivity for red wavelength; this is similar to the response reported previously. ${ }^{(1)}$ In the 400 to

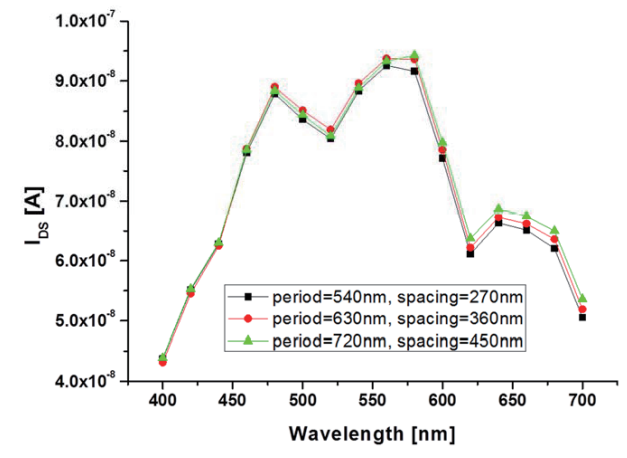

(a)

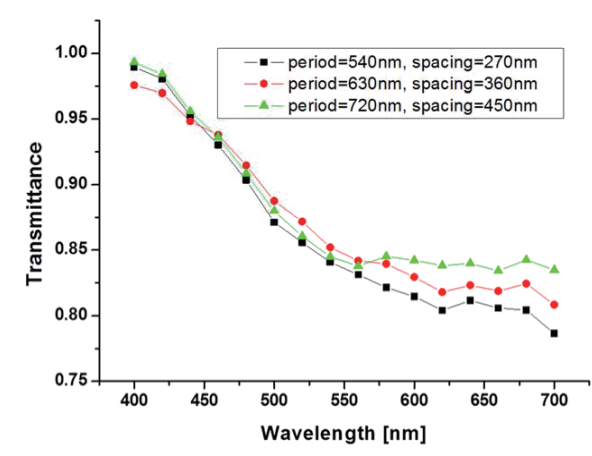

(b)

Fig. 7. (Color online) (a) Drain current and (b) transmittance of gate/body-tied MOSFET-type photodetector with 1D metal grid.

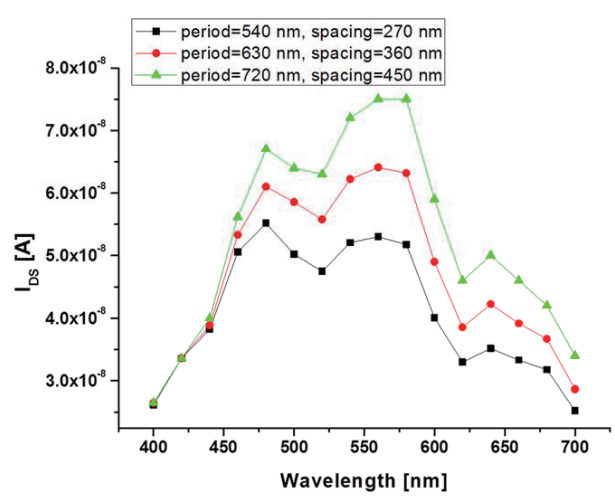

(a)

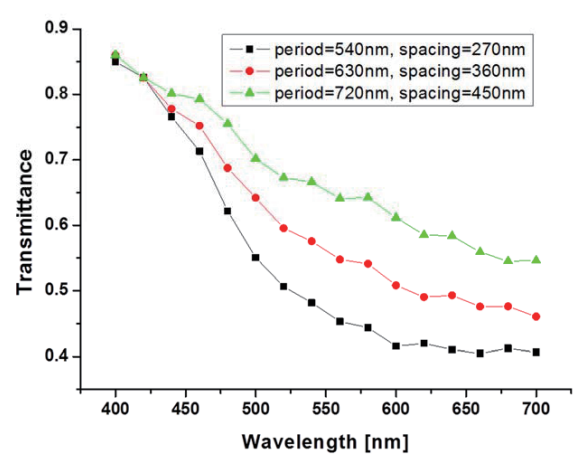

(b)

Fig. 8. (Color online) (a) Drain current and (b) transmittance of gate/body- tied MOSFET-type photodetector with 2D metal grid. 
$700 \mathrm{~nm}$ range, the gate/body-tied photodetector with a 1D metal grid pattern shows low selectivity but high transmittance, whereas the gate/body-tied photodetector with a 2D metal grid pattern shows high selectivity but low transmittance. Moreover, the spectral drain current of the proposed photodetector is larger than that of the conventional $n+$ / p-sub photodiode reported in previous works. ${ }^{(1,6)}$

Thus, we were able to confirm that placing a metal grid pattern on top of a gate/bodytied photodetector can significantly affect its spectral transmittance. In the future, the spectral transmittance of the proposed photodetector with additional signal processing will be able to improve wavelength selectivity.(1)

\section{Conclusions}

A new design of a gate/body-tied MOSFET-type photodetector with a patterned metal grid was proposed and demonstrated. This new design improved the sensitivity and wavelength selectivity of the conventional photodetector. The proposed photodetector was fabricated using standard 1-poly, 4-metal, 0.18- $\mu$ m CMOS technology, without postprocessing. The $1 \mathrm{D}$ and $2 \mathrm{D}$ metal grids were fabricated using the metal-1 layer, which is closest to the photodetector. The metal grid patterns were designed with three different spacings and periodicities for the purpose of comparison. These patterns had periods/spacings of 540/270,630/360, and 720/450 $\mathrm{nm}$. The gate/body-tied MOSFETtype photodetector amplified the photocurrent due to a positive feedback mechanism. The responsivity of the gate/body-tied photodetector was mroe than 1000-fold that of the conventional $\mathrm{n}+/ \mathrm{p}$-sub photodiode with the same photodetection area. To demonstrate wavelength selectivity, the spectral drain current and transmittance of the proposed photodetector were measured. The transmittance of the gate/body-tied MOSFETtype photodetector with the 1D metal grid increased when the period/spacing of the metal grid increased. Similarly, the transmittance of the gate/body-tied MOSFET-type photodetector with the 2D metal grid increased when the period/spacing of the metal grid increased.

From the above results, we confirmed that the proposed gate/body-tied MOSFETtype photodetector with a metal grid pattern can control the spectral transmittance with a high responsivity. By employing advanced processes and by using finer metal width and spacing, the gate/body-tied photodetector with a metal grid pattern could replace the CMOS color filters.

\section{Acknowledgements}

This research was supported by the Basic Science Research Program of the National Research Foundation of Korea (NRF), funded by the Ministry of Education (No. 2012R1A1A2007512); the BK21 Plus project funded by the Ministry of Education, Korea (21A20131600011); and the Integrated Circuit Design Education Center (IDEC), Korea. 


\section{References}

1 P. B. Catrysse and B. A. Wandell: J. Opt. Soc. Am. A 20 (2003) 2293.

2 K. A. Parulski: IEEE Trans. Electron Devices 32 (1985) 1381.

3 P. Dillon, D. Lewis and F. Kaspar: IEEE Trans. Electron Devices 25 (1978) 102.

4 H. Nabeyama: IEEE Trans. Consum. Electron. 27 (1981) 40.

5 A. J. Blanksby and M. J. Loinaz: IEEE Trans. Electron Devices 47 (2000) 55.

6 F. Yang and A. H. Titus: Electron. Lett. 43 (2007) 1279.

7 A. J. Makynen, J. T. Kostamovaara and T. E. Rahkonen: IEEE Trans. Instrum. Meas. 43 (1994) 489.

8 J.-H. Park, S.-H. Seo, I.-S. Wang, H.-J. Yoon, J.-K. Shin, P. Choi, Y.-C Jo and H. Kim: Sens. Mater. 15 (2003) 361.

9 W. Zang and M. Chan: IEEE Trans. Electron Dev. 48 (2001) 1097.

10 Y. Nagamune, T. Noda, Y. Ohno, Y. Arakawa, H. Sakaki and M. Watanabe: Jpn. J. Appl. Phys. 36 (1997) 1955.

11 H. F. Ghaemi, T. Thio, D. E. Grupp, T. W. Ebbesen and H. J. Lezec: Phy. Rev. B 58 (1998) 6779.

12 C. Genet and T. W. Ebbesen: Nature 445 (2007) 39.

13 H. A. Bethe: Phys. Rev. 66 (1944) 163.

14 T. W. Ebbesen: Nature 391 (1998) 667. 